\title{
Containment Studies of Transgenic Mosquitoes in Disease Endemic Countries: The Broad Concept of Facilities Readiness
}

\author{
M. Megan Quinlan,, Josephine Birungi,,3 Mamadou B. Coulibaly, Abdoulaye Diabaté, \\ Luca Facchinelli, Wolfgang Richard Mukabana,,8 James Mutuku Mutunga,,* \\ Tony Nolan, ${ }^{1}$ Peter Raymond, ${ }^{10}$ and Sékou F. Traoré ${ }^{4}$
}

\begin{abstract}
Genetic strategies for large scale pest or vector control using modified insects are not yet operational in Africa, and currently rely on import of the modified strains to begin preliminary, contained studies. Early involvement of research teams from participating countries is crucial to evaluate candidate field interventions. Following the recommended phased approach for novel strategies, evaluation should begin with studies in containment facilities. Experiences to prepare facilities and build international teams for research on transgenic mosquitoes revealed some important organizing themes underlying the concept of "facilities readiness," or the point at which studies in containment may proceed, in sub-Saharan African settings. First, "compliance" for research with novel or non-native living organisms was defined as the fulfillment of all legislative and regulatory requirements. This is not limited to regulations regarding use of transgenic organisms. Second, the concept of "colony utility" was related to the characteristics of laboratory colonies being produced so that results of studies may be validated across time, sites, and strains or technologies; so that the appropriate candidate strains are moved forward toward field studies. Third, the importance of achieving "defensible science" was recognized, including that study conclusions can be traced back to evidence, covering the concerns of various stakeholders over the long term. This, combined with good stewardship of resources and appropriate funding, covers a diverse set of criteria for declaring when "facilities readiness" has been attained. It is proposed that, despite the additional demands on time and resources, only with the balance of and rigorous achievement of each of these organizing themes can collaborative research into novel strategies in vector or pest control reliably progress past initial containment studies.
\end{abstract}

Keywords: biosafety, compliance, containment, insectary, mosquitoes, transgenic

\footnotetext{
${ }^{1}$ Centre for Environmental Policy, Imperial College London, Ascot, United Kingdom.

${ }^{2}$ Uganda Virus Research Institute (UVRI), Entebbe, Uganda.

${ }^{3}$ International Livestock Research Institute, Nairobi, Kenya.

${ }^{4}$ Université des Sciences, des Techniques et des Technologies de Bamako, Bamako, Mali.

${ }^{5}$ Institut de Recherche en Sciences de la Santé (IRSS)/Centre Muraz, Bobo Dioulasso, Burkina Faso.

${ }^{6}$ Department of Vector Biology, Liverpool School of Tropical Medicine, Liverpool, United Kingdom.

${ }^{7}$ School of Biological Sciences, University of Nairobi, Nairobi, Kenya.

${ }^{8}$ Science for Health, Nairobi, Kenya.

${ }^{9}$ International Center of Insect Physiology and Ecology (ICIPE), Mbita Point, Kenya.

${ }^{10}$ Donald Danforth Plant Science Center, St. Louis, Missouri.

*Current affiliation: Department of Biological Sciences, Mount Kenya University, Thika, Kenya.
}

(C) M. Megan Quinlan et al. 2018 Published by Mary Ann Liebert, Inc. This article is available under the Creative Commons License CC-BY-NC (http://creativecommons.org/licenses/by-nc/4.0). This license permits non-commercial use, distribution and reproduction in any medium, provided the original work is properly cited. Permission only needs to be obtained for commercial use and can be done via RightsLink. 


\section{Introduction}

$\mathbf{L}$ IVESTOCK AND HORTICULTURAL insect pests and some disease vectors have been suppressed or eradicated using irradiated, or in one case chemically induced, sterile insect release in several African countries over recent decades (Klassen and Curtis 2007). ${ }^{\dagger}$ Genetic strategies employing novel symbiosis, transgenesis, genome editing (e.g., with TALENs or CRISPR-Cas9), or other modification for insect control, however, are still in the research phase in Africa and are not yet delivering the benefits of operational control programs. For the present, use of these novel approaches in field programs in sub-Saharan Africa would require import of the technology to establish colonies of transgenic strains, if not for producing them.

Several scientific expert groups have recommended a stepwise approach to genetic strategies beginning with studies in containment facilities, before field cages or confined field studies are employed to answer further research questions that may support open field release (NAPPO 2007, Benedict et al. 2008, AHTEG 2010, James et al. 2010, EFSA 2011, WHO/ TDR, and FNIH 2014, National Academies of Sciences, Engineering, and Medicine 2016).

A component of the work leading up to the Target Malaria project $^{\S}$ was to develop the infrastructure and capacity for containment studies in African settings. Containment studies of modified insects take place within authorized facilities designed to restrict exposure of people and the environment to the study organism. Unlike biological containment for materials known to be infectious (OECD 2007, CDC 2009, Homer et al. 2013), containment for initial studies of transgenic organisms is due to lack of experience or knowledge at the early phases of research or technology development. In the case of novel mosquito strains, a phased approach also allows progressively more realistic conditions in which hypothesisdriven studies on safety, quality, and efficacy would be difficult to complete in field conditions (Ferguson et al. 2008), in part due to higher recapture rates than in open field studies, and thus more accessible data (Madakacherry et al. 2014).

Early involvement of research teams in countries with the targeted insect population is a critical part of this phased approach to evaluation of potential field interventions. This is especially true for interventions in public health that require some form of community consent, in this case targeting

\footnotetext{
${ }^{\dagger}$ These pilot and field programs have taken place at various times in Ethiopia, Kenya (Lounibos 2003, McDonald et al. 1977), Libya, Mauritius (Seewooruthun et al. 2000), Morocco, Nigeria (Oladunmade et al. 1990), Reunion, Senegal (Dicko et al. 2014), South Africa, Sudan, Tanzania (Vreysen et al. 2000) and Tunisia (Chakroun et al. 2015) against various spp. of tsetse fly, New World screwworm (Lindquist et al. 1992), Mediterranean fruit fly (Enkerlin 2005), date or carob moth, codling moth, diamond back moth, and vector spp. of mosquitoes (Anopheles arabiensis and Aedes aegypti).

TALEN are transcription activator-like effector nucleases and CRISPR are clustered regularly interspaced short palindromic repeats. While this article focuses on transgenic mosquitoes, most of the commentary applies to other methods employed in genetic strategies, although risks may vary.

§Target Malaria (www.targetmalaria.org) is a nonprofit research consortium committed to developing an innovative approach to reduce the number of malaria vector mosquitoes and therefore the transmission of the disease, complementary to other control methods. Some of those in the research consortium reported in this study continue to work together in this project.
}

malaria vector species (Chu et al. 2014, Kolopack et al. 2015). We describe broad principles that supported our research consortium's preparation for containment studies of transgenic mosquitoes in four African settings.

\section{Preparing for Containment Studies}

Within this context, the collaboration described in this study - a discovery laboratory team moving toward an international research consortium-began working toward what we refer to as "facilities readiness" to prepare for containment studies with transgenic mosquitoes in Burkina Faso, Mali, Uganda, and Kenya. An important aspect of our preparation was to discuss and agree on some fundamental concepts. As with any good project management, early discussions about containment studies included agreements on data collection, analysis, and ownership; presentation and publication of results; and harmonized use of key terms. Early discussions with regulatory authorities also ensured that plans would be made within the national regulatory frameworks. (The process of establishing two-way dialog with stakeholders to achieve codevelopment of the technology is described in forthcoming publications.)

Early on we concluded that neither "Good Laboratory Practice" at the level of certification, nor a single international standard, such as ISO/IEC 17025 (ISO 2005), fits with the studies planned. There is no "one standard" that covers all aspects of the objectives of preparing containment facilities to study modified insects. The Arthropod Containment Guidelines developed over time by the American Committee of Medical Entomology (ACME/ASTMH 2003), however, proved invaluable guidance for preparations. These guidelines describe that Arthropod Containment Levels (ACLs) related more appropriately to containment of arthropods (including mosquitoes) than is found in descriptions of Biosafety Levels based on microbiological and pathogenic hazards (Scott 2005, Tabachnick 2006, CDC 2009). The guidelines recommend ACL-2 for uninfected transgenic mosquitoes. ACL-2 indicates several design features and stringent procedures to physically isolate the study organism from the open environment and guard against release of any viable life stages from the facility. The key components of ACL-2 and specific corresponding measures are shown in Figure 1.

Although the guidelines were useful, there was no single standard or even term that gave the sense of reaching the point in preparations when an import permit could be sought. Biosafety implies the management of risks associated with the use of "any living organism that possesses a novel combination of genetic material obtained through the use of modern biotechnology" (UNEP 2000). However, biosafety is a term used differently in various contexts, and translated differently among languages (Waage and Mumford 2008, WHO and CBD 2015, Quinlan et al. 2016a). We adopted a new term, "facilities readiness," to express three underpinning concepts: "compliance," "colony utility," and "defensible science." Each concept supported our efforts toward being confident and reliable researchers of a novel technology, beginning with studies in containment.

\section{Compliance}

We approached the concept of "compliance" as the fulfillment of all legislative and regulatory requirements-and 


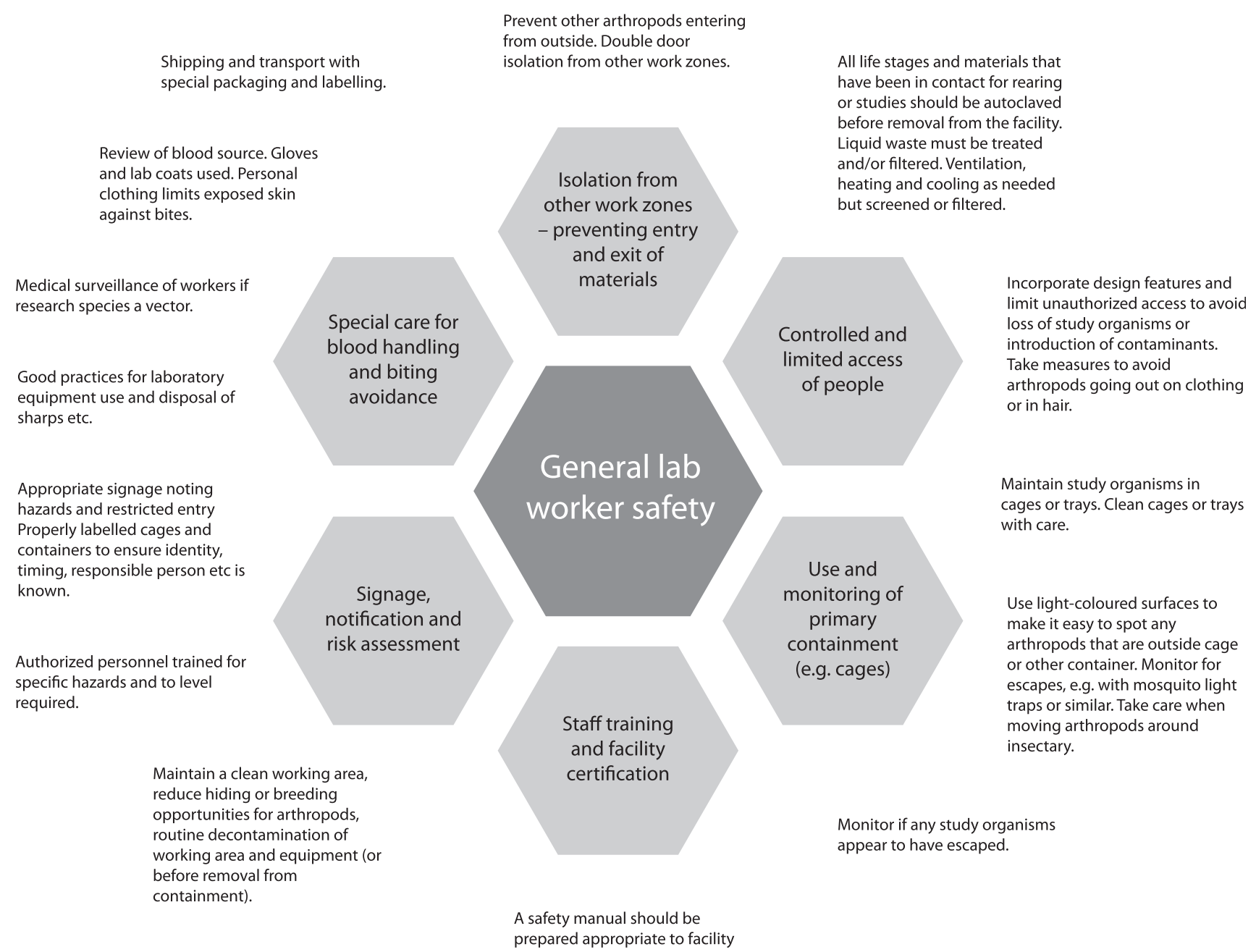

FIG. 1. Key components of arthropod containment level 2 containment and example measures relevant to transgenic mosquitoes.

the underlying intentions of the requirements - for studies on novel or non-native living organisms; in this case, transgenic malaria vector mosquitoes. For research on and use of transgenic organisms, a separate biosafety framework was established in the majority of the signatory countries to implement the Cartagena Protocol on Biosafety to the Convention on Biological Diversity (UNEP 2000).** This often operates through the National Biosafety Authority (NBA) responsible to make decisions under the national biosafety framework, for example the Agence Nationale de Biosécurité and the Comité National de Biosécurité in Burkina Faso and Mali, respectively. We talked about intentions of regulations because biosafety frameworks generally were developed in the context of crop biotechnology and aspects of the regulations may not apply to mosquitoes, whereas the underlying

**A separate framework is not a requirement of the Protocol, but advocacy groups and considerable funding encouraged this approach. Some countries are considering a more comprehensive riskbased framework that could address other sources of biological risks, particularly in light of emerging techniques and technologies that are not strictly covered by the Protocol and generally have encountered a gap or uncertainty in the regulatory pathway. principles remain relevant. Once a specific study or import approval is granted, compliance includes the fulfillment of any terms and conditions of approval by the NBA for facilities or studies therein. This may be at the time of the import or studies, or subsequently, as indicated by the regulatory authorities.

In addition to regulations under the biosafety framework, there are multiple laws, decrees, and guidelines relating to research, public health interventions, biotechnology businesses, and so forth, before importing or studying a novel mosquito strain, including: research permits for originating and/or recipient institutes; shipping requirements for biological material; material transfer agreement specifications of the country or exporting/importing institute; intellectual property control; regulations on collecting, rearing, and releasing mosquitoes that are a potential vector of human disease; and non-native species restrictions.

Because the NBA often works through a committee comprised of representatives from the various ministries and authorities, such additional requirements will probably be highlighted in the course of reviewing an application for import or studies of a transgenic organism. These other regulations may not be rationalized with a biosafety framework, 
however, because the Cartagena Protocol came into force much later than many other national regulatory requirements. Therefore, we chose to obtain advice from legal and regulatory experts in the region before submitting an application, to identify any gaps in compliance with national regulations during our preparations. Local or subregional requirements might also be imposed (Beech and Miller 2014), although most of these will be related to construction of a facility.

The requirements for hosting a containment facility (availability of land for the construction, connection to water and electricity services, and existing emergency and security services) are generally linked to an institution and a prerequisite to applying for a research or import permit; permits for field studies also require a local hosting institution. Under the biosafety framework in many countries, there would need to be an Institutional Biosafety Committee (IBC), with its membership notified to the country's NBA. In Kenya, for example, the IBC serves as a communication liaison to consult with the NBA in preparation of applications for containment studies. Once authorization is granted, the IBC may be further involved to perform internal audits and document overall compliance with the terms and conditions of authorization. This may be scheduled to target certain critical activities, or be conducted without notice as "spot audits" to monitor ongoing activities. If the principal investigator (PI) of the study is a member of the IBC, it may facilitate answering questions, but the PI should avoid conflict of interests in that role. Additional members of an IBC may be needed to add expertise on mosquito biology, vectored disease control, and public health research.

While some countries may certify a facility to a specific containment level and allow annual reporting of studies, other countries require that an application detailing each study to be conducted in the facility be submitted to the NBA for review and approval. Whatever the framework, our experience is that, to a large degree, ongoing monitoring of compliance with terms and conditions of approvals relies heavily on the researchers themselves, or the IBC. This is an approach followed in parts of Europe as well. This responsibility further indicates the usefulness of proper documentation of monitoring and compliance, or "defensible science," hereunder discussed, to justify the trust placed in the research institution for this role. Some aspects of compliance are also relevant to "colony utility," including maintaining the integrity of study organisms in terms of genetic identity as well as not losing any material under study.

Compliance may include formal notification of activities and public engagement, under the terms of regulations. While public engagement on a regulatory decision is less often required for work inside containment (versus field trials), good practice may include providing opportunities for public education to support more informed participation as the technology moves into the next stages (Quinlan et al. 2016b), as well as stakeholder engagement aimed at genuine two-way dialog, and not only compliance.

A final aspect of compliance is good stewardship of the project resources, financial transparency, and progress reporting. Detailed requirements for this aspect generally appear in the funding or collaboration agreement.

\section{Colony Utility}

A perhaps obvious, but not often articulated, concept is that of "colony utility." We defined this as maintaining and monitoring the identity and quality of mosquitoes being produced so that results of studies may be compared across time (through generations, and over months or years), sites (various laboratories), and strains or products. Comparable results are not always harmonized. A collaborative effort has to contend with differences between facilities and work styles of already-existing research teams that may not have previously worked together. Natural variation within the same species of Anopheles influenced by the wild-type stock being used could cause variation in study results. Such variations may affect subsequent field outcomes as well as early phase study results (Yeap et al. 2013).

We decided to systematize the culturing methods to the degree possible while allowing for local procurement of diet, despite diet being a significant factor (Gilles et al. 2011). Although the vector species under study require a set range in temperature (Bayoh and Lindsay 2003, 2004) and humidity in the insectary, attempting to replicate with precision a narrow range of all of the environmental conditions in each containment facility was rejected as unnecessarily onerous and costly; studies requiring this could be done in more expensive facilities with environmental chambers. Instead, each site aims for similar output quality indicators (Mumford et al., 2018). The most manageable yet informative indicators were selected over the course of 2 years' discussion and trials and are now incorporated into a bespoke insectary database covering every generation produced.

Even when aligning quality assurance, diverse facilities may be working with field-caught populations that naturally vary in some phenotypic if not always genetic characteristics (Paton et al. 2013). To establish the relationship among these indicators for each population colonized for studies, a benchmarking procedure was established (Valerio et al. 2016a). Each laboratory population may be compared on different diet regimes by measuring wing length and development rate. This initial benchmarking will tell the research team whether significant differences are inherent to the various populations, whether they are related to something other than diet and larval density, and whether study results may be analyzed without adjustment across sites. Consistent differences could be expressed in calibrations to adjust findings across sites. Once benchmarking is completed, it is important for routinely used laboratory consumables such as diet to be procurable locally, and from a consistent source, for ease of laboratory management and standardization.

A further factor in colony utility, which was noted for compliance as well, is whether one actually knows with certainty the identity of the mosquitoes used in any given study, at any given location, for any given time. To address this, our consortium adhered to a two-step colony establishment procedure following field collection. Field-caught gravid females are kept separate past egg laying, and sample progeny from the same family are submitted for species confirmation using PCR to eliminate the small possibility of hybrids in the colony. Routine sampling every few generations continues to confirm identity of the colonies, although in most cases facilities are single use and possibility of strain contamination is low. Monitoring for free-flying mosquitoes, with identification at genus level to determine if entering from outside or from the research colony, is recorded in the same database for quality indicators. This could be increased to species identification of all free fliers if the distinction is not clear. Another way to 
ensure cross-site harmonization is to agree a single method of polymerase chain reaction (PCR), or alternative validated method for genetic identification, such as loop mediated isothermal amplification (LAMP), for species and strain identity, and to compare interpretation of results. To maintain "defensible science," all of these steps toward colony utility should be documented for clear understanding into the future.

The utility factor is, of course, closely linked to enabling researchers to adequately answer questions, or verify predictions and assumptions (Valerio et al. 2016b), to then properly select the best transgenic strain to take forward into a multilaboratory study or confined field trial, with introgressions into each local wild-type strain. The expense of later-stage studies would prohibit progressing numerous candidates.

\section{Defensible Science}

We understand "defensible science" as providing evidence of maintaining identity of the biological materials; agreeing and managing the documentation of compliance, study designs, methods, and results; and maintaining training and staffing records. All of these should be accomplished in a manner that can be traced back to each transgenic insertion event, but also each step in product development, such as shipping strains. The objective is to provide clear, repeatable, reliable, and accessible evidence appropriate to the interests and concerns of various stakeholders, over the long term. This concept also is not entirely new (Guindo et al. 2012 describe documentation for preparation for clinical trials), although not always articulated sufficiently in research consortia. This requires staff who are well trained and committed to achieving a standard beyond what is generally practiced. However, given the anticipated use of the transgenic or otherwise-modified mosquito itself as the intervention technology, the necessity of documentation of the colonies in use increases dramatically from the level of data compilation undertaken for research alone.

At the beginning of the collaboration, agreement was sought on what type, timing (in relation to other events and in frequency), and level of documentation would be needed to achieve defensible science. A simple template was elaborated in Excel ${ }^{\circledR}$, the wild-type colony record, to record and organize details about the establishment of colonies from the target populations of mosquito vector species (Quinlan et al. 2016c). Similarly, a certificate of analysis was developed that covers all aspects considered important for material transfer, confirmation, and maintenance of research strains. These certificates are in addition to the conventional material transfer documentation required for shipping mosquito eggs in secure, tracked, and environmentally maintained conditions. Data on samples from the field population, the field-caught colony established in the laboratory, and the transgenic strain were included in the insectary database. The key quality indicators to show the maintenance of colony utility were also incorporated into the database (Mumford et al., in this issue), to be recorded with the setup of each cage. A standard operating procedure (SOP) was prepared to harmonize use of laboratory notebooks, while SOPs on other methods and study protocols instruct the teams on data management, templates, and electronic backup.

The choice of parameters reflected not only the need for answering study questions that would allow researchers to move on to the next phase, but also covered data useful for regulatory purposes and to populate or validate models used for internal risk assessments and management. Our aim is that all decisions during development of the ultimate goal technologies or product(s) are documented and the reasoning for the decisions are clear; methodologies are always described; and all data can be traced back to laboratory notebooks, field notes, or original data templates for at least 5 years into the future. Finally, when hands-on training is complete or the actual studies begin, use of data templates and timely data review and analysis further support work standards and expectations on a daily basis. Shared access to data and timely analysis can provide further insurance against misunderstandings or disjointed work plans more efficiently than further meetings or discussions after the foundation for collaboration has been laid. The defensible science aspect of the preparation of the facilities and the research teams is illustrated in Figure 2.

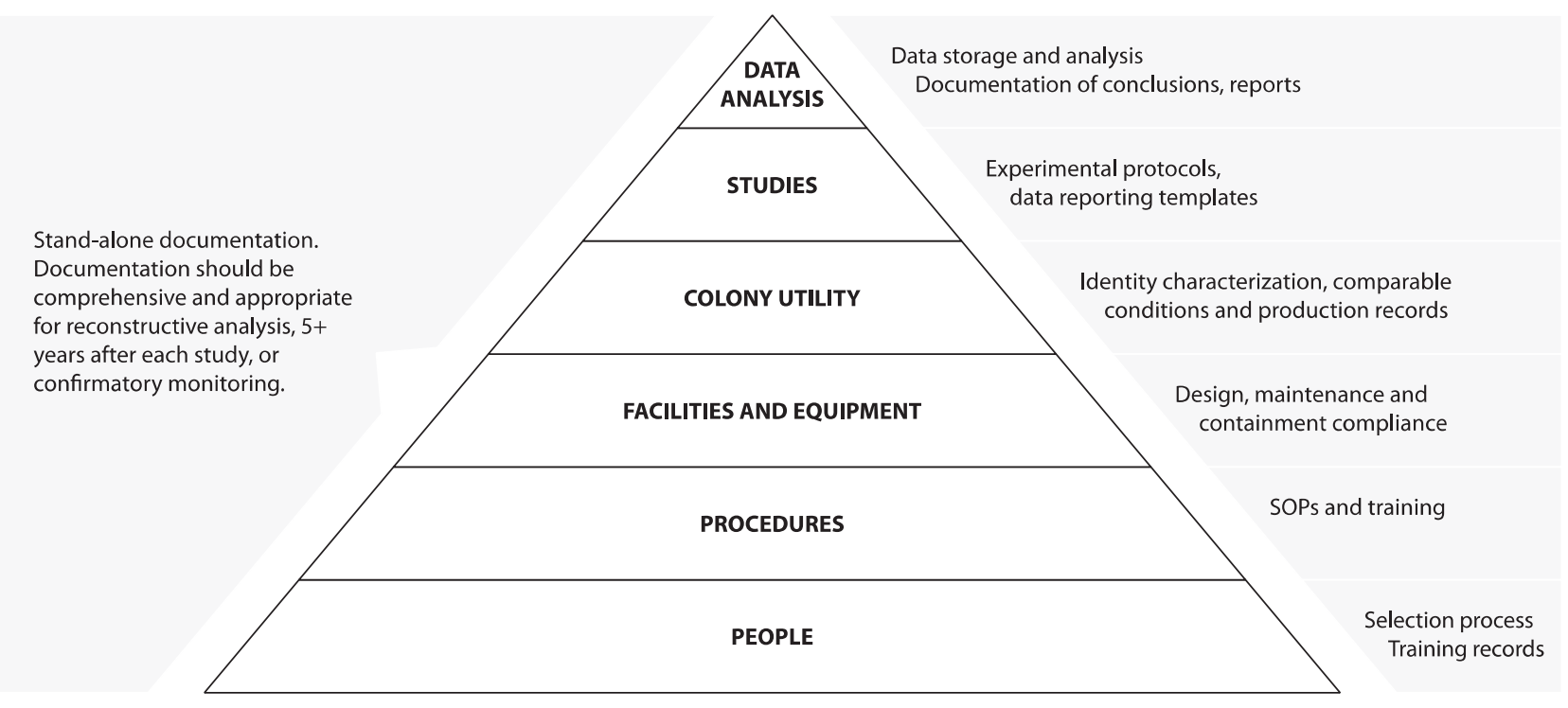

FIG. 2. Components of facilities readiness preparations leading to reliable study results and defensible science. 
The documentation of these components does not leave them static, but rather each adjustment made during operations should be documented and explained. Choices made in terms of procedures, equipment, and even facility design should be reviewed over time to ensure their effectiveness to reach the targeted objectives in terms of containment and colony utility. This also means that such objectives should be clearly described at the very beginning, based on a detailed risk analysis, thereby tying defensible science back to compliance and colony utility.

\section{Discussion}

We describe in this study, and in another article focusing on operational details (Quinlan et al., 2018), a process of preparing for studies in containment and achieving "facilities readiness," before import of transgenic mosquitoes to a subSaharan Africa site. The process described is an example of moving from a discovery laboratory team to an international partnership with African research teams and facilities. There are a number of design features and procedures in an authorized facility to ensure achieving a suitable level, such as ACL-2 (Fig. 1). However, it is through shared learning and recognition that, as highlighted in Figure 2, people are the foundation of such a preparation that real progress was made. Reaching agreement on appropriate indicators for colony utility and the necessary documentation for defensible science takes time, yet can build trust and prevent later misunderstandings. Planning for and monitoring compliance also requires rigorous attention, resources, and time. If the practices learned from this experience are followed, however, the preparation process in other locations or for other genetic strategies may be facilitated.

Careful and considered preparations for the broad concepts of compliance, colony utility, and defensible science have laid a foundation for future progression of promising technologies from laboratory to field. It is proposed that, despite the additional demands on time and resources, only with the balance of and rigorous achievement of each of these organizing themes can collaborative research into novel strategies for vector control reliably progress past initial containment studies.

\section{Acknowledgments}

This collaborative work was largely funded by a grant from the Foundation for the National Institutes of Health (FNIH) through the Vector-Based Control of Transmission: Discovery Research (VCTR) program of the Grand Challenges in Global Health (GCGH) initiative of the Bill \& Melinda Gates Foundation (BMGF). All authors confirm there is no conflict of interest regarding reporting on this experience or carrying out the work, and that the funder did not limit their ability to develop the approach described. The authors also wish to thank Rebecca Murphy for editorial support and John Mumford for article review.

\section{Author Disclosure Statement}

No competing financial interests exist.

\section{References}

ACME/ASTMH (American Committee of Medical Entomology/ American Society of Tropical Medicine and Hygiene).
Arthropod Containment Guidelines, version 3.1. Vector Borne Zoonotic Dis 2003; 3:61-98.

AHTEG (Ad Hoc Technical Expert Group on Risk Assessment and Risk Management). Risk assessment of living modified mosquitoes. In: Convention on Biological Diversity, ed. Guidance on Risk Assessment of Living Modified Organisms. Montreal, Canada: Secretariat of the Convention on Biological Diversity, United Nations Environmental Programme, 2010:80-94.

Bayoh MN, Lindsay SW. Effect of temperature on the development of the aquatic stages of Anopheles gambiae sensu stricto (Diptera: Culicidae). Bull Entomol Res 2003; 93:375-381.

Bayoh MN, Lindsay SW. Temperature-related duration of aquatic stages of the Afrotropical malaria vector mosquito Anopheles gambiae in the laboratory. Med Vet Entomol 2004; 18:174-179.

Beech C, Miller T. Risk analysis and the regulation of transgenic insects. In: Benedict MQ, ed. Transgenic Insects: Techniques and Applications. Wallingford, UK: CABI, 2014:319-336.

Benedict M, D'Abbs P, Dobson S, Gottlieb M, et al. Guidance for contained field trials of vector mosquitoes engineered to contain a gene drive system: Recommendations of a scientific working group. Vector Borne Zoonotic Dis 2008; 8:127-166.

CDC (Centers for Disease Control and Prevention). Biosafety in Microbiological and Biomedical Laboratories, 5th edn. Atlanta, GA: CDC, 2009.

Chakroun S, Skander M, Lebdi KG. Sterile insect technique against date moth Ectomyelois ceratoniae Zeller 1881, in Tunisia: Research of gamma radiation sterilising dose. Int $\mathbf{J}$ Sci Res Eng Technol 2015; 3:55-62.

Chu KM, Jayaraman S, Kyamanywa P, Ntakiyiruta G. Building research capacity in Africa: Equity and global health collaborations. PLoS Med 2014; 11:e1001612.

Dicko AH, Lancelot R, Seck MT, Guerrini L, et al. Using species distribution models to optimize vector control in the framework of the tsetse eradication campaign in Senegal. Proc Natl Acad Sci U S A 2014; 111:10149-10154.

EFSA (European Food Safety Authority). Genetically modified animals. 2011. Available at www.efsa.europa.eu/en/topics/ topic/gmanimals.htm

Enkerlin WR. Impact of fruit fly control programmes using sterile insect technique. In: Dyck VA, Hendrichs J, Robinson AS, eds. Sterile Insect Technique: Principles and Practice in Area-wide Integrated Pest Management. Dordrecht, Netherlands: Springer, 2005:651-676.

Ferguson HM, Ng'habi KR, Walder T, Kadungula D, et al. Establishment of a large semi-field system for experimental study of African malaria vector ecology and control in Tanzania. Malar J 2008; 7:158.

Gilles JRL, Lees RS, Soliban SM, Benedict MQ. Densitydependent effects in experimental larval populations of Anopheles arabiensis (Diptera: Culicidae) can be negative, neutral, or overcompensatory depending on density and diet levels. J Med Entomol 2011; 48:296-304.

Guindo MA, Shott JP, Saye R, Diakité ML, et al. Promoting good clinical laboratory practices and laboratory accreditation to support clinical trials in sub-Saharan Africa. Am J Trop Med Hyg 2012; 86:573-579.

Homer LC, Alderman TS, Blair HA, Brocard A-S, et al. Guidelines for biosafety training programs for workers assigned to BSL-3 research laboratories. Biosecur Bioterror 2013; 11:10-19.

ISO (International Organization for Standardization). ISO/IEC 17025: 2005. General requirements for the competence of testing and calibration laboratories. 2005. Available at www .iso.org/standard/39883.html 
James AA, Mumford JD, James SL, Touré YT, eds. Progress and Prospects for the Use of Genetically Modified Mosquitoes to Inhibit Disease Transmission. Geneva, Switzerland: World Health Organization, 2010.

Klassen W, Curtis CF. History of the sterile insect technique. In: Dyck VA, Hendrichs J, Robinson AS, eds. Sterile Insect Technique: Principles and Practice in Area-wide Integrated Pest Management. Dordrecht, Netherlands: Springer, 2005:3-36.

Kolopack PA, Parsons JA, Lavery JV. What makes community engagement effective? Lessons from the eliminate dengue program in Queensland Australia. PLoS Negl Trop Dis 2015; 9:e0003713.

Lindquist DA, Abusowa M, Hall MJR. The New World screwworm fly in Libya: A review of its introduction and eradication. Med Vet Entomol 1992; 6:2-8.

Lounibos LP. Genetic control trials and ecology of Aedes aegypti at the Kenyan coast. In: Takken W, Scott TW, eds. Ecological Aspects for Application of Genetically Modified Mosquitoes. Wageningen, Netherlands: Kluwer, 2003:34-43.

Madakacherry O, Lees RS, Gilles JRL. Aedes albopictus (Skuse) males in laboratory and semi-field cages: Release ratios and mating competitiveness. Acta Trop 2014; 132:S124-S129.

McDonald PT, Hausermann W, Lorimer N. Sterility introduced by release of genetically altered males to a domestic population of Aedes aegypti at the Kenya coast. Am J Trop Med Hyg 1977; 26:553-561.

Mumford JD, Leach AW, Benedict MQ, Facchinelli L, et al. Maintaining quality of candidate strains of transgenic mosquitoes for studies in containment facilities in disease endemic countries. Vector Borne Zoonotic Dis 2018; 18:31-38.

NAPPO (North American Plant Protection Organization). RSPM 27. Guidelines for importation and confined field release of transgenic arthropods in NAPPO member countries. 2007. Available at www.nappo.org/english/standards-andprotocols/regional-phytosanitary-standards-rspms

National Academies of Sciences, Engineering, and Medicine. Gene Drives on the Horizon: Advancing Science, Navigating Uncertainty, and Aligning Research with Public Values. Washington, DC: The National Academies Press, 2016.

OECD (Organisation for Economic Co-operation and Development). OECD best practice guidelines for biological resource centers. 2007. Available at www.oecd.org/sti/biotech/ 38777417.pdf

Oladunmade MA, Feldmann U, Takken W, Tenabe SO, et al. Eradication of Glossina palpalis palpalis (Robineau-Desvoidy) (Diphera: Glosinidae) from agropastoral land in central Nigeria by means of the insect sterile technique. In: Offori ED, Van der Vloedt AMV, eds. Sterile Insect Technique for Tsetse Control and Eradication. Vienna, Austria: IAEA, 1990:5-23.

Paton D, Touré M, Sacko A, Coulibaly MB, et al. Genetic and environmental factors associated with laboratory rearing affect survival and assortative mating but not overall mating success in Anopheles gambiae sensu stricto. PLoS One 2013; 8:e82631.

Quinlan MM, Alden J, Habbel F, Murphy R. The biosecurity approach: A review and evaluation of its application by FAO, internationally and in various countries. 2016a. Available at www.ippc.int/static/media/files/irss/2016/09/09/Review_of_ biosecurity_approaches_FINAL_report.pdf

Quinlan MM, Smith J, Layton R, Keese, P, et al. Experiences in engaging the public on biotechnology advances and regulation. Front Bioeng Biotechnol 2016b; 4:3.
Quinlan MM, Sanou R, Namountougou M, Yagoure B, et al. Providing a pedigree for future genetic strategy technology. Poster presented at Pan-African Mosquito Control Association (PAMCA) Third Annual Conference, Lagos, Nigeria, 2016c.

Quinlan MM, Mutunga J, Diabaté A, Namountougou M, et al. Studies of transgenic mosquitoes in disease endemic countries: Preparation of containment facilities. Vector Borne Zoonotic Dis 2018; 18:21-30.

Scott TW. Containment of arthropod disease vectors. ILAR J 2005; 46:53-71.

Seewooruthun SI, Permalloo S, Gungah B, Soonnoo AR, et al. Eradication of an exotic fruit fly from Mauritius. In: Tan KH, ed. Area-Wide Control of Fruit Flies and Other Insect Pests. Pulau Pinang: Penerbit Universiti Sains Malaysia, 2000:389-394.

Tabachnick W. Laboratory containment practices for arthropod vectors of human and animal pathogens. Lab Anim (NY) 2006; 35:28-33.

UNEP (United Nations Environment Programme). Cartagena protocol on biosafety to the convention on biological diversity. 2000. Available at https://bch.cbd.int/protocol/text

Valerio L, Collins CM, Lees RS, Benedict MQ. Benchmarking vector arthropod culture: An example using the African malaria mosquito, Anopheles gambiae (Diptera: Culicidae). Malar J 2016a; 15:262.

Valerio L, North A, Collins CM, Mumford JD, et al. Comparison of model predictions and laboratory observations of transgene frequencies in continuously-breeding mosquito populations. Insects 2016b; 7:47.

Vreysen MJB, Saleh KM, Ali MY, Abdulla AM, et al. Glossina austeni (Diptera: Glossinidae) eradicated on the island of Unguja, Zanzibar, using the sterile insect technique. J Econ Entomol 2000; 93:123-135.

Waage JK, Mumford JD. Agricultural biosecurity. Philos Trans R Soc London B Biol Sci 2008; 363:863-876.

WHO and CBD (World Health Organization and Convention on Biological Diversity). Infectious diseases. In: WHO, ed. Connecting Global Priorities: Biodiversity and Human Health: A State of Knowledge Review. Geneva, Switzerland: World Health Organization, 2015:130-149.

WHO/TDR and FNIH (World Health Organization/Special Programme for Research and Training in Tropical Diseases and Foundation for the National Institutes of Health). Guidance framework for testing genetically modified mosquitoes. 2014.

Yeap HL, Endersby NM, Johnson PH, Ritchie SA, et al. Body size and wing shape measurements as quality indicators of Aedes aegypti mosquitoes destined for field release. Am $\mathbf{J}$ Trop Med Hyg 2013; 89:78-92.

Address correspondence to: M. Megan Quinlan Centre for Environmental Policy Imperial College London 1.09 Hamilton Building Silwood Park Campus Ascot, SL5 7PY Berkshire United Kingdom

E-mail: m.quinlan@imperial.ac.uk 